\title{
Comparação entre simulação e sistema real de vibração com dois graus de liberdade
}

\author{
Comparison between simulation and real two degrees of freedom vibration system
}

\author{
Alexandre J. Bühler ${ }^{* 1}$, Matheus Lago ${ }^{1}$, Vitor G. Bergamim¹, Matheus R. Deves ${ }^{1}$ \\ ${ }^{1}$ Instituto Federal do Rio Grande do Sul, Campus Farroupilha, Farroupilha, RS, Brasil
}

\begin{abstract}
Recebido em 25 de Outubro de 2018. Revisado em 28 de Fevereiro de 2019. Aceito em 18 de Abril de 2019
Na engenharia, compreender corretamente os elementos que compõem os sistemas vibratórios e a física envolvida nestes é fundamental no projeto de estruturas e máquinas, por exemplo. Embora em muitos casos, no estudo de sistemas vibratórios não seja possível analisar o sistema somente com um grau de liberdade, sistemas com dois graus ou mais são muito pouco abordados em livros de Física Geral. Este artigo traz a fundamentação teórica para a análise de sistemas de dois graus de liberdade e apresenta um sistema construído com duas massas e três molas vibrando na direção do campo gravitacional da Terra. Para este sistema foi realizada uma simulação por meio do software Modellus que foi comparada com dados reais da vibração do sistema construido, obtidos por meio do software Tracker. Os dados medidos foram analisados utilizando curvas de ajuste de acordo com o modelo físico proposto e por meio de transformada rápida de Fourier. Em ambos os casos, os resultados encontrados para os dois modos de vibração estão de acordo com o previsto pela teoria.
\end{abstract}

Palavras-chave: Vibrações Mecânicas; Tracker; Modellus.

\begin{abstract}
In engineering, to correctly understand the elements presented in vibratory systems and the physics involved in them is paramount for designing of structures and machines, for example. Although in many cases, in the study of vibratory systems it is not possible to analyze the system only with one degree of freedom, systems with two degrees or more are very little approached in General Physics books. This article presents the theoretical basis for the analysis of systems of two degrees of freedom and presents a system constructed with two masses and three springs vibrating in the direction of the gravitational field of the Earth. For this system, a simulation was carried out using the Modellus software, which was compared with the actual system vibration data obtained through the Tracker software. The measured data were analyzed using fitting curves according to the proposed physical model and by means of Fast Fourier Transform. In both cases, the found results for the two modes of vibration are in according with the predicted by theory.
\end{abstract}

Keywords: Mechanical Vibrations; Tracker; Modellus.

\section{Introdução}

A análise de sistemas vibracionais é muito importante na Física e Engenharia. Nesta última, vibrações estão presentes no projeto de máquinas, estruturas e veículos, por exemplo. As aplicações do estudo de vibrações são diversas, entre as quais podem-se destacar a análise de curvas de ressonância para evitar colapsos em estruturas e a busca de relações adequadas de amortecimento, rigidez e massa para proporcionar segurança e conforto em veículos.

Quando abordada em livros de Física Geral, os sistemas vibracionais possuem apenas um grau de liberdade (gdl). Em muitos casos, mesmo que seja óbvio que o sistema em estudo possua mais de um gdl, uma aproximação para apenas um grau pode ser suficiente. Entretanto existem diversas outras situações onde não se pode negligenciar

*Endereço de correspondência: alexandre.buhler@farroupilha.ifrs.edu.br os demais gdl do sistema em estudo. Embora o estudo de sistemas com mais de um gdl seja fundamental em diversas aplicações na Física e na Engenharia, tais sistemas são, em geral, omitidos dos livros de Física Geral e, consequentemente, não comentados em aula.

É evidente que associar o estudo da teoria de sistemas vibracionais com atividades experimentais tem o potencial de auxiliar fortemente no aprendizado do aluno, principalmente em caráter multidisciplinar. Entretanto, laboratórios para estudo de vibrações mecânicas são onerosos e, portanto, nem sempre disponíveis. Existem algumas alternativas muito mais econômicas e que podem fornecer excelentes resultados, principalmente para finalidades didáticas. Em 1], por exemplo, é usado um sistema de aquisição de dados baseado na plataforma Arduino. O sensor de posição usado para medir a vibração é um sensor de ultrasson e a taxa de aquisição de dados é de $248 \mathrm{~Hz}$. Essa taxa proporcionou, no caso em estudo, 
uma aquisição de dados satisfatória para a análise de um sistema de vibração com frequência de $44 \mathrm{~Hz}$.

Em [2] é apresentado o estudo de um sistema de vibração do tipo shear building com três gdl . Neste estudo, o sistema de aquisição de dados também faz uso da plataforma Arduino, porém o sistema é também excitado com uma força harmônica externa. Na análise no domínio de frequência efetuado neste trabalho os autores confrontam as frequências naturais medidas com as previamente calculadas. A determinação das frequências naturais é feita através dos picos de ressonância e os resultados se mostraram bastante próximos com o previsto pela teoria.

Entretanto, dependendo do tipo de vibração a ser analisada e das simplificações impostas ao modelo físico aplicado, as diferenças entre os valores calculados e medidos podem ser significativas. Em um trabalho publicado por Mahoney e Nathan [3], são analisados os quatro primeiros modos de vibração para o eixo $x$ (eixo perpendicular ao campo gravitacional) e $z$ (eixo paralelo ao campo gravitacional) de uma escada de alumínio na vertical. Algumas simplificações são impostas ao modelo utilizado e os autores acreditam que estas podem ser responsáveis por diferenças da ordem de $10 \%$ entre os valores medidos e calculados. Ainda assim, a aplicabilidade da metodologia proposta por $[3]$ apresenta uma interessante aplicabilidade didática em aulas de vibrações mecânicas.

Outra possibilidade de aquisição de dados de custo muito baixo é através do software Tracker. O Tracker é um software livre que trabalha com a linguagem Java para análise e modelagem de movimentos gravados em vídeo. Em um trabalho escrito por Bonventi e Aranha 4 o Tracker é usado para aquisição de dados para um sistema de oscilação amortecida (pêndulo físico). Neste caso a taxa de aquisição é definida pela número de quadros por segundo que o aparelho usado para gravar o vídeo possui. No trabalho de Bonventi e Aranha foi usado um smartphone (aquisição em cerca de 24 quadros por segundo). Essa taxa se mostrou consideravelmente satisfatória na análise da oscilação do pêndulo físico de frequência de aproximadametne $0,9 \mathrm{~Hz}$.

Em um trabalho publicado por Shiki et al. 5 é analisado um sistema com dois graus de liberdade cuja a oscilação é medida usando acelerômetros e com vídeo, analisado pelo software Tracker. Esse sistema possui duas massas de valor igual e três molas também de valor igual, o que de acordo com a literatura $[6]$ fornece autovetores iguais a 1 e -1(esse conceito será é discutido na seção Fundamentação teórica). São apresentados os períodos de oscilação correspondentes aos dois modos de vibração experimentais, porém não é realizada uma comparação com os valores teóricos, que não são apresentados em [5].

Este trabalho apresenta um estudo de um sistema de 2 gdl consistindo de 3 molas com diferentes constantes de rigidez $(k)$ e 2 massas diferentes $(m)$. Este mesmo sistema foi simulado usando o software Modellus, porém sem considerar os efeitos de amortecimento inerentes a oscilação em meio fluido. Essa decisão foi tomada uma vez que para baixas velocidades o efeito do amortecimento do sistema no ar é muito pequeno e a frequência amortecida é muito próxima a frequência natural. O sistema de vibração foi também medido usando o software Tracker e os dados de amplitude e tempo de ambas as massas foram analisados usando o software Curve Expert 7 para obtenção dos parâmetros dentro do modelo físico proposto (o mesmo usado no Modellus). Os mesmos dados também foram analisados usando Transformada Rápida de Fourier. Os resultados para as frequências dos modos de vibração encontrados por ambos os métodos ficaram bastante próximos dos obtidos pela simulação do Modellus e, portanto, em acordo com a teoria.

\section{Fundamentação teórica}

Sistemas de dois gdl são os que necessitam de duas coordenadas independentes para descrever o seu movimento [6].

A Fig. 1 representa um sistema de dois gdl, onde temse duas massas, $m_{1}$ e $m_{2}$, e três molas, com constantes elásticas $k_{1}, k_{2}$ e $k_{3}$, sem amortecimento e sem a aplicação de forças externas (com exceção da força peso das massas).

É possível demonstrar que, quando um sistema oscilatório vibra na vertical, a força peso em cada massa do

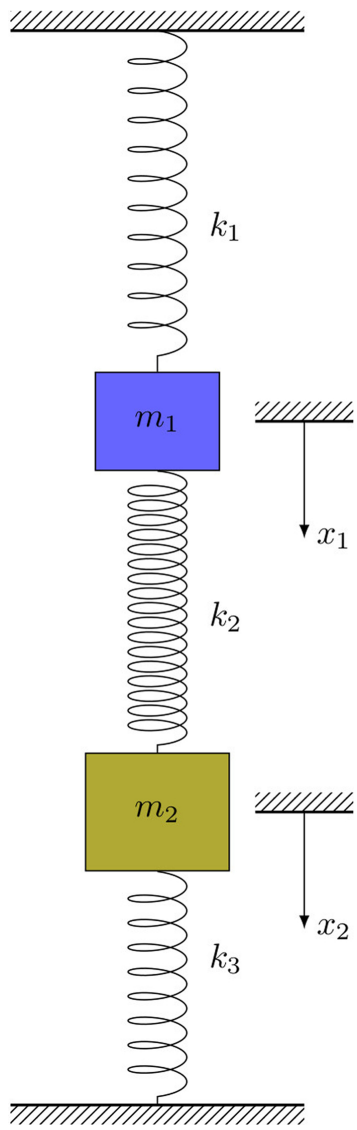

Figura 1: Sistema vibracional com dois graus de liberdade. 
sistema apenas altera a posição de equilíbrio de um valor equivalente a $m g / k$ [8], [9]. Dessa forma, as frequências naturais de vibração do sistema não sofrem qualquer alteração. Visto que o principal objetivo deste trabalho é medir e comparar as frequências naturais de um sistema real com os valores teóricos, a dedução das equações do movimento realizada a seguir não leva em conta o campo gravitacional.

Entretanto, para que um experimento como tal seja realizado, é importante que sejam levados alguns pontos em consideração.

- O deslocamento ocasionado pelo peso de cada massa não pode ser tal que, ao vibrar, os elos das molas inferiores possam se tocar entre si. Isso afetaria o comportamento experimental do sistema oscilatório.

- Deve-se ter cuidado para que o peso de cada massa não ocasione um deslocamento grande o suficiente de forma que a mola possa operar fora de sua região de linearidade.

- A massa de cada mola deve ser muito inferior à massa de cada corpo acoplado às molas do sistema. Caso esse critério não seja respeitado, o modelo físico adotado deve ser diferente levando em conta o que se entende por massa equivalente do sistema. Para este trabalho, as massas de cada mola são muito inferiores às massas oscilatórias, de forma que a modelagem aplicada trata as molas como tendo massas desprezíveis.

- Deve ter o cuidado de por o sistema a oscilar com condições iniciais que evitem a vibração do sistema na direção perpendicular ao eixo das molas. Por exemplo, se for conferida uma posição inicial a uma das massas que possua alguma componente perpendicular ao campo gravitacional, então o sistema irá oscilar com mais graus de liberdade e isso certamente afetará o comportamento físico do sistema.

Para que o sistema oscilatório apresentado na Fig. 1] ser modelado matematicamente, duas equações, uma para cada massa, são necessárias. Essas equações são denominadas Equações Diferenciais Acopladas(EDA). Cada equação envolve todas as variáveis do sistema.

Para encontrar as EDA pode-se fazer uso da segunda Lei de Newton analisando o diagrama de corpo livre do sistema, conforme mostra a Fig. 2 .

A partir da análise do diagrama de corpo livre da Fig. 2 e negligenciando as forças peso, obtém-se a equação (11) e a equação (2).

$$
\begin{aligned}
& m_{1} \ddot{x}_{1}(t)+\left(k_{1}+k_{2}\right) x_{1}(t)-k_{2} x_{2}(t)=0 \\
& m_{2} \ddot{x}_{2}(t)-k_{2} x_{1}(t)+\left(k_{2}+k_{3}\right) x_{2}(t)=0
\end{aligned}
$$

Uma vez obtidas as equações que determinam o movimento das massas, é necessário resolvê-las. Para isso, soluções harmônicas correspondentes às equações (3) e
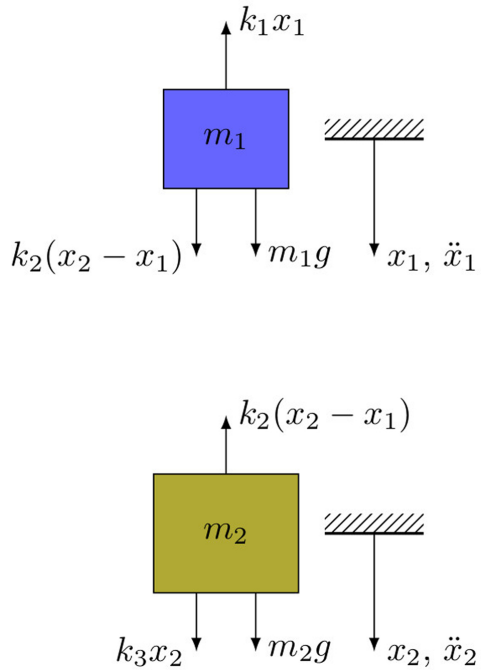

Figura 2: Diagrama de corpo livre do sistema com dois graus de liberdade.

(4) são assumidas para cada coordenada, onde as variáveis $X_{1}$ e $X_{2}$ são constantes que determinam os valores de amplitude máxima de $x_{1}(t)$ e de $x_{2}(t)$ e $\varphi$ é o ângulo de fase.

$$
\begin{aligned}
& x_{1}(t)=X_{1} \cos (\omega t+\varphi) \\
& x_{2}(t)=X_{2} \cos (\omega t+\varphi)
\end{aligned}
$$

Substituindo as equações (3) e (4) em (1) e (2), encontrase:

$$
\left\{X_{1}\left[-m_{1} \omega^{2}+\left(k_{1}+k_{2}\right)\right]-k_{2} X_{2}\right\} \cos (\omega t+\varphi)=0
$$

$\left\{X_{2}\left[-m_{2} \omega^{2}+\left(k_{2}+k_{3}\right)\right]-k_{2} X_{1}\right\} \cos (\omega t+\varphi)=0$

Para que as equações 5 e 6 sejam satisfeitas, a parte oscilante de cada equação deve ser zero para qualquer tempo. Para que isso ocorra, a seguinte afirmação deve ser verdadeira:

$$
\cos (\omega t+\varphi)=0
$$

Dessa forma, o sistema resultante das equações (5) e (6) é dado por:

$$
\begin{aligned}
& X_{1}\left[-m_{1} \omega^{2}+\left(k_{1}+k_{2}\right)\right]-k_{2} X_{2}=0 \\
& X_{2}\left[-m_{2} \omega^{2}+\left(k_{2}+k_{3}\right)\right]-k_{2} X_{1}=0
\end{aligned}
$$

Analisando-se o sistema, é possível perceber que a solução trivial existe para quando $X_{1}=X_{2}=0$ porém, quando isso acontece, não existe movimento oscilatório. Dessa forma, para que a solução não seja trivial, a determinante dos coeficientes $X_{1}$ e $X_{2}$ deve ser zero: 
$\operatorname{det}\left[\begin{array}{cc}\left\{-m_{1} \omega^{2}+\left(k_{1}+k_{2}\right)\right\} & -k_{2} \\ -k_{2} & \left\{-m_{2} \omega^{2}+\left(k_{2}+k_{3}\right)\right\}\end{array}\right]=0$

(9)

ou:

$$
\begin{aligned}
& \left(m_{1} m_{2}\right) \omega^{4}-\left\{\left(k_{1}+k_{2}\right) m_{2}+\left(k_{2}+k_{3}\right) m_{1}\right\} \omega^{2} \\
& +\left\{\left(k_{1}+k_{2}\right)\left(k_{2}+k_{3}\right)-k_{2}^{2}\right\}=0
\end{aligned}
$$

A solução da equação (10) fornece as frequências $\omega_{1}$ e $\omega_{2}$, que são as duas frequências naturais do sistema. Essas frequências são também chamadas de autovalores e são apresentados na equação (11).

$$
\omega_{1,2}=\sqrt{\left(\frac{\left(k_{1}+k_{2}\right) m_{2}+\left(k_{2}+k_{3}\right) m_{1}}{2 m_{1} m_{2}}\right) \pm \sqrt{\left[\left(\frac{\left(k_{1}+k_{2}\right) m_{2}+\left(k_{2}+k_{3}\right) m_{1}}{2 m_{1} m_{2}}\right)^{2}-2\left(\frac{\left(k_{1}+k_{2}\right)\left(k_{2}+k_{3}\right)-k_{2}^{2}}{m_{1} m_{2}}\right)\right]}}
$$

Agora, torna-se necessário encontrar os valores de $X_{1}$ e $X_{2}$. Entretanto, os valores de $X_{1}$ e $X_{2}$ vão variar dependendo da frequência que está sendo analisada. Portanto, serão utilizados os seguintes índices para diferencia-los: $X_{1}^{(1)}$ e $X_{2}^{(1)}$ para quando o sistema vibra com a frequência $\omega_{1}$ e (primeiro modo de vibração) $X_{1}^{(2)}$ e $X_{2}^{(2)}$ para quando o sistema vibra com a frequência $\omega_{2}$ (segundo modo de vibração).

Rearranjando o sistema das equações (7) e (8) de forma a encontrar a razão entre $X_{1}^{(1)}$ e $X_{2}^{(1)}$ e entre $X_{1}^{(2)}$ e $X_{2}^{(2)}$, tem-se:

$$
\begin{aligned}
& r_{1}=\frac{X_{2}^{(1)}}{X_{1}^{(1)}}=\frac{k_{2}}{-m_{2} \omega_{1}^{2}+\left(k_{2}+k_{3}\right)} \\
& r_{2}=\frac{X_{2}^{(2)}}{X_{1}^{(2)}}=\frac{k_{2}}{-m_{2} \omega_{2}^{2}+\left(k_{2}+k_{3}\right)}
\end{aligned}
$$

As razões $r_{1}$ e $r_{2}$ são os autovetores correspondentes aos autovalores. Um autovetor representa a proporção entre os deslocamentos dos graus de liberdade para cada frequência de ressonância ou autovalor. Em um trabalho de conclusão de curso escrito por Nishi Ueta $[10$ é feita uma análise de um sistemas de vibração com três graus de liberdade e é possível encontrar uma explicação mais detalhada sobre autovetores e autovalores em sistemas de vibração mecânica.

Uma vez conhecidas as razões $r_{1}$ e $r_{2}$, é possível escrever os vetores dos modos normais de vibração:

$$
\begin{aligned}
\vec{X}^{(1)} & =\left[\begin{array}{l}
X_{1}^{(1)} \\
X_{2}^{(1)}
\end{array}\right]=\left[\begin{array}{c}
X_{1}^{(1)} \\
r_{1} X_{1}^{(1)}
\end{array}\right] \\
\vec{X}^{(2)} & =\left[\begin{array}{l}
X_{1}^{(2)} \\
X_{2}^{(2)}
\end{array}\right]=\left[\begin{array}{c}
X_{1}^{(2)} \\
r_{2} X_{2}^{(2)}
\end{array}\right]
\end{aligned}
$$

Com os modos de normais de vibração definidos é possível escrever a solução da vibração livre em função do tempo:

$$
\begin{aligned}
& \vec{x}^{(1)}(t)=\left[\begin{array}{l}
x_{1}^{(1)}(t) \\
x_{2}^{(1)}(t)
\end{array}\right]=\left[\begin{array}{c}
X_{1}^{(1)} \cos \left(\omega_{1} t+\varphi_{1}\right) \\
r_{1} X_{1}^{(1)} \cos \left(\omega_{1} t+\varphi_{1}\right)
\end{array}\right] \\
& \vec{x}^{(2)}(t)=\left[\begin{array}{l}
x_{1}^{(2)}(t) \\
x_{2}^{(2)}(t)
\end{array}\right]=\left[\begin{array}{c}
X_{1}^{(2)} \cos \left(\omega_{2} t+\varphi_{2}\right) \\
r_{2} X_{1}^{(2)} \cos \left(\omega_{2} t+\varphi_{2}\right)
\end{array}\right]
\end{aligned}
$$

Sabendo-se que a soma das soluções de uma Equação Diferencial é, também uma solução, é possível rearranjar as equações (16) e (17), obtendo-se:

$$
\begin{aligned}
& x_{1}(t)=x_{1}^{(1)}(t)+x_{1}^{(2)}(t)=X_{1}^{(1)} \cos \left(\omega_{1} t+\varphi_{1}\right) \\
& +X_{1}^{(2)} \cos \left(\omega_{2} t+\varphi_{2}\right)
\end{aligned}
$$

e

$$
\begin{aligned}
& x_{2}(t)=x_{2}^{(1)}(t)+x_{2}^{(2)}(t)=r_{1} X_{1}^{(1)} \cos \left(\omega_{1} t+\varphi_{1}\right) \\
& +r_{2} X_{1}^{(2)} \cos \left(\omega_{2} t+\varphi_{2}\right)
\end{aligned}
$$

Todavia, ainda é necessário determinar as variáveis $X_{1}^{(1)}, X_{1}^{(2)}, \varphi_{1}, \varphi_{2}$, que podem ser encontradas a partir das condições iniciais impostas ao sistema. Como as Equações Diferenciais que descrevem os movimentos das massas são de segunda ordem, duas condições iniciais para cada massa são necessárias. As condições serão as posições iniciais $\left(x_{1}(0)=x_{1}^{(i)}\right.$ e $\left.x_{2}(0)=r_{i} x_{i}^{(i)}\right)$ e as velocidades iniciais $\left(\dot{x_{1}}(0)\right.$ e $\dot{x_{2}}(0)$.

Substituindo as condições iniciais nas equações (18) e (19), obtem-se:

$$
\begin{aligned}
X_{1}^{(1)} \cos \varphi_{1} & =\left\{\frac{r_{2} x_{1}(0)-x_{2}(0)}{r_{2}-r_{1}}\right\} \\
X_{1}^{(2)} \cos \varphi_{2} & =\left\{\frac{-r_{1} x_{1}(0)+x_{2}(0)}{r_{2}-r_{1}}\right\}
\end{aligned}
$$




$$
\begin{aligned}
X_{1}^{(1)} \sin \varphi_{1} & =\left\{\frac{-r_{2} x_{1} \dot{(}(0)+x_{2} \dot{(}(0)}{\omega_{1}\left(r_{2}-r_{1}\right)}\right\} \\
X_{1}^{(2)} \sin \varphi_{2} & =\left\{\frac{r_{1} x_{1}(0)-x_{2} \dot{(}(0)}{\omega_{2}\left(r_{2}-r_{1}\right)}\right\}
\end{aligned}
$$

Resolvendo o sistema de quatro equações e quatro variáveis, obtem-se os parâmetros $X_{1}^{(1)}, X_{1}^{(2)}, \varphi_{1}$ e $\varphi_{2}$ que devem ser inseridos na equação (18) e equação (19) forncendo as equações dinâmicas para $m_{1}$ e $m_{2}$.

$$
\begin{gathered}
X_{1}^{(1)}=\frac{1}{r_{2}-r_{1}}\left\{\left[r_{1} x_{1}(0)-x_{2}(0)\right]^{2}\right. \\
\left.+\left[\frac{-r_{2} \dot{x_{1}}(0)+\dot{x_{2}}(0)}{\omega_{1}^{2}}\right]^{2}\right\}^{\frac{1}{2}} \\
X_{1}^{(2)}=\frac{1}{r_{2}-r_{1}}\left\{\left[-r_{1} x_{1}(0)+x_{2}(0)\right]^{2}\right. \\
\left.+\left[\frac{r_{1} \dot{x_{1}}(0)-\dot{x_{2}}(0)}{\omega_{2}^{2}}\right]^{2}\right\}^{\frac{1}{2}} \\
\varphi_{1}=\arctan \left\{\frac{-r_{2} \dot{x_{1}}(0)+\dot{x_{2}}(0)}{\omega_{1}\left[r_{2} x_{1}(0)-x_{2}(0)\right]}\right\} \\
\varphi_{2}=\arctan \left\{\frac{r_{1} \dot{x_{1}}(0)-\dot{x_{2}}(0)}{\omega_{2}\left[-r_{1} x_{1}(0)+x_{2}(0)\right]}\right\}
\end{gathered}
$$

Para que o sistema vibre no primeiro modo de vibração é necessário que a segunda frequência natural seja anulada nas equações (24) e (25). Isso é possível se as as velocidades iniciais forem nulas e se a posição inicial de $m_{2}$ for igual a $r_{1}$ vezes a posição inicial de $m_{1}$. Para operar no segundo modo de vibração a condição necessária é a de velocidades iniciais nulas e posição inicial de $m_{2}$ igual a $r_{2}$ vezes a posição inicial de $m_{1}$. Com quaisquer outras condições iniciais o sistema irá vibrar com ambos os modos de vibração ao mesmo tempo, sendo a intensidade de cada um definida pelas condições iniciais.

\section{Sistema real com dois graus de liberdade}

O sistema vibratório construido para análise neste trabalho é constituído de duas massas feitas de nylon e três molas de aço. A massa de menor valor, denominada de $m_{1}$ foi usinada de forma a adquirir a forma de um cilindro com diâmetro e altura de $72 \mathrm{~mm}$. Já a massa de maior valor $\left(m_{2}\right)$, também cilíndrica, tem diâmetro de $102 \mathrm{~mm}$ e altura de $66 \mathrm{~mm}$. As molas são interligadas a duas bases de mdf com dimensões de $260 \mathrm{~mm}$ x 260 $\mathrm{mm} \times 215 \mathrm{~mm}$ e entre si por meio de três molas de aço. Para dar sustentação ao sistema, as bases de mdf foram conectadas a três barras de aço rosqueadas com diâmetro de $10 \mathrm{~mm}$. A distância entre as bases de mdf é de 840 mm. Essa distância garante com que cada mola fique estendida o suficiente para que em nenhum momento da vibração exista a colisão entre os elos das molas, o que aferia o movimento oscilatório do sistema. A Fig. 3 apresenta uma imagem do sistema de $2 \mathrm{gdl}$ desenvolvido com as principais dimensões em verde sobre a figura.

Para a determinação das constantes elásticas das molas foram usados quatro blocos de massas distintas. Essas foram medidas e presas a cada mola separadamente. Com a mola suspensa em uma extremidade fixa e com a outra extremidade sustentando o bloco. Foram feitas medidas com a mola em repouso e posteriormente foram medidas as deflexões das molas com as massas. Para as quatro situações foram anotados os valores do peso com a respectiva variação de comprimento da mola. Um gráfico relacionando a força aplicada e a deformação na mola foi gerado, por meio do qual a inclinação da curva corresponde a constante elástica da mola. Esse procedimento considera que as molas obedecem a lei de Hooke.

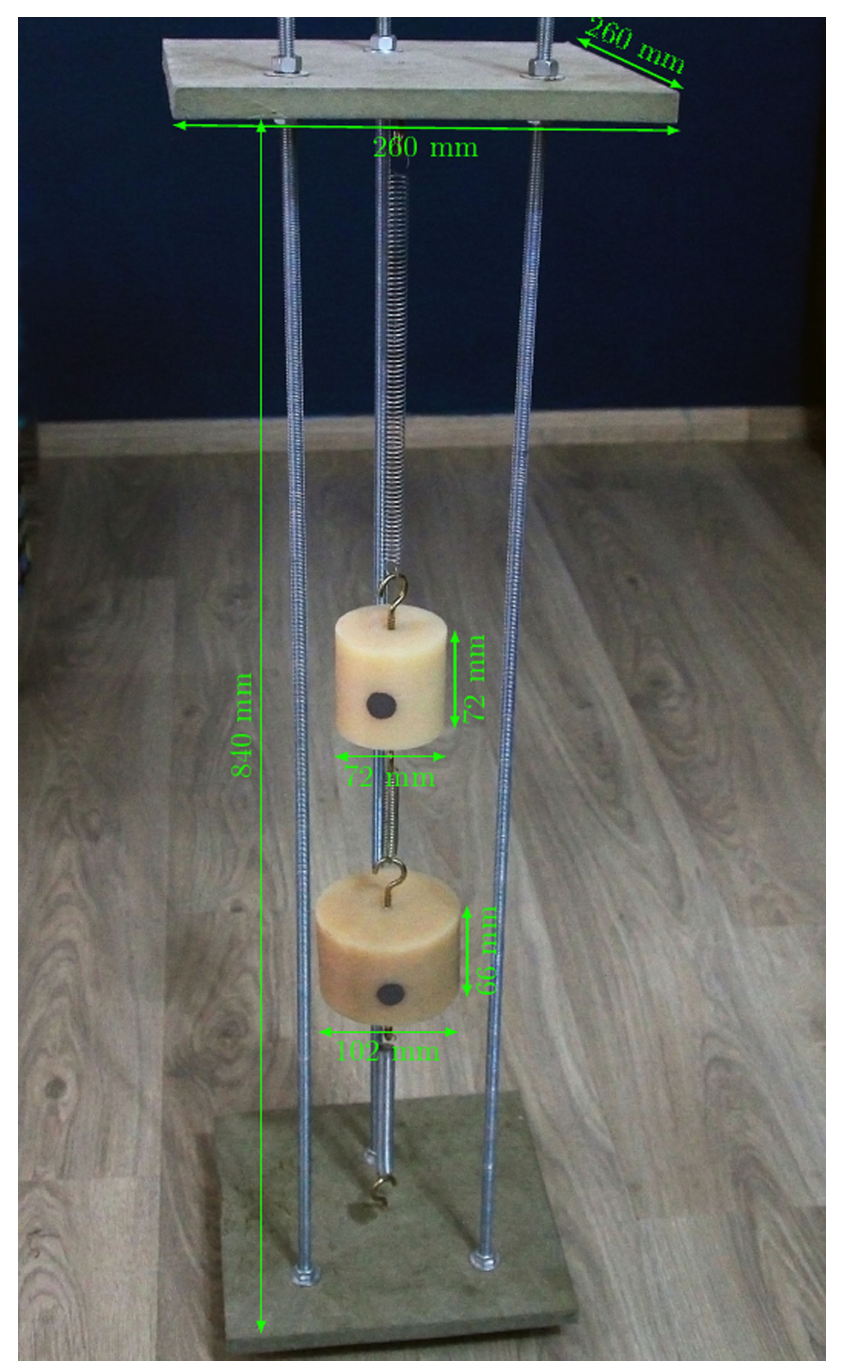

Figura 3: Sistema vibratório de dois graus de liberdade construido para este trabalho. 
A medição das massas foi feita com uma balança eletrônica. A incerteza desse instrumento foi admitida como sendo $\pm 0,001 \mathrm{~kg}$ (resolução da medida). As medidas das massas do sistema oscilatório forneceram $\mathrm{m}_{1}=0,341 \pm$ $0,001 \mathrm{~kg}$ e $\mathrm{m}_{2}=0,624 \pm 0,001 \mathrm{~kg}$.

Levando em consideração a altitude e latitude do local (29ํํㄹ de latitude e $780 \mathrm{~m}$ de altitude) onde foram realizadas as medidas, a aceleração gravitacional calculada foi de $9,79 \mathrm{~m} / \mathrm{s}^{2}$ [11]. Logo, a incerteza obtida na medida das forças é a multiplicação da incerteza da balança pela aceleração da gravidade, que resulta em aproximadamente 0,01 N. Para medir a deflexão das molas foi usado uma régua milimétrica. A incerteza admitida para esse instrumento foi de $\pm 0,0005 \mathrm{~m}$ (metade da resolução da medida).

As constantes elásticas foram calculadas para cada uma das quatro medidas, usando a lei de Hooke. A partir disso, foi calculado o desvio padrão dessa amostra de pontos. Esse resultado foi definido como a incerteza de cada constante elástica. Nas Figuras 4,5] 6, são apresentados gráficos de força $\times$ deflexão de cada mola, com as suas respectivas incertezas mencionadas (incerteza da força e incerteza da deformação da mola). Também é mostrado o desvio padrão da rigidez das molas. Os valores medidos das molas ficaram em $\mathrm{k}_{1}=132,3 \pm 0,3 \mathrm{~N} / \mathrm{m}, \mathrm{k}_{2}=59,4$ $\pm 0,7 \mathrm{~N} / \mathrm{m} \mathrm{e} \mathrm{k}_{3}=50,2 \pm 0,6 \mathrm{~N} / \mathrm{m}$.

\section{Simulação com o software Modellus}

O software Modellus é uma ferramenta bastante versátil e relativamente simples de ser usada no ensino de Física. A grande vantagem do uso do Modellus é, sem dúvida, a possibilidade de interpretar as equações de movimento por meio de animações. Inúmeros autores têm feito o uso do Modellus como ferramenta no ensino de Física. Como exemplo, pode-se destacar [12, 13 e [14 que utiliza o Modellus para a conceitualização de derivada.

$\mathrm{Na}$ plataforma do Modellus foram usadas as equações apresentadas na Fundamentação Teórica, que permitiram

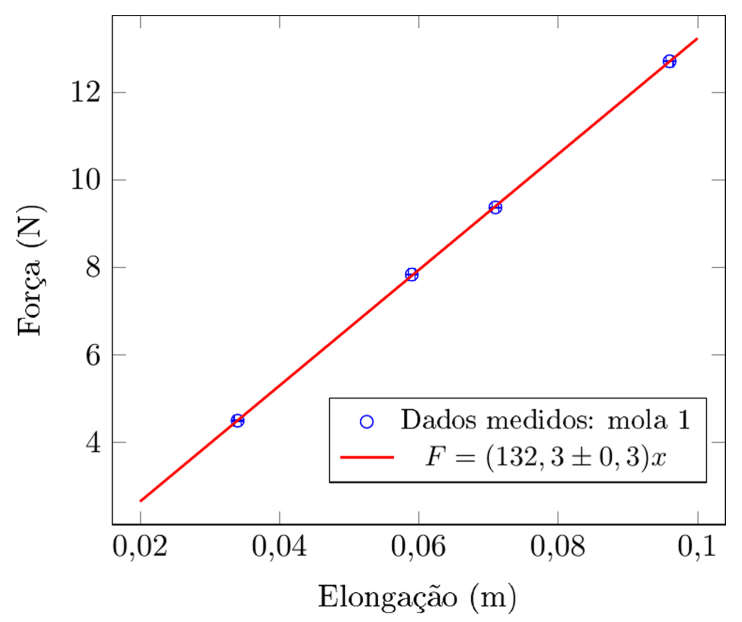

Figura 4: Constante de Rigidez da Mola 1

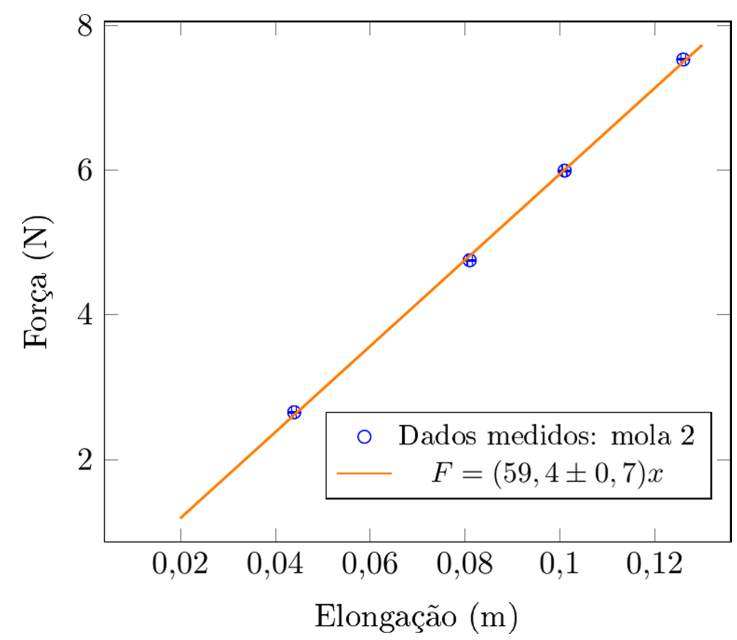

Figura 5: Constante de Rigidez da Mola 2

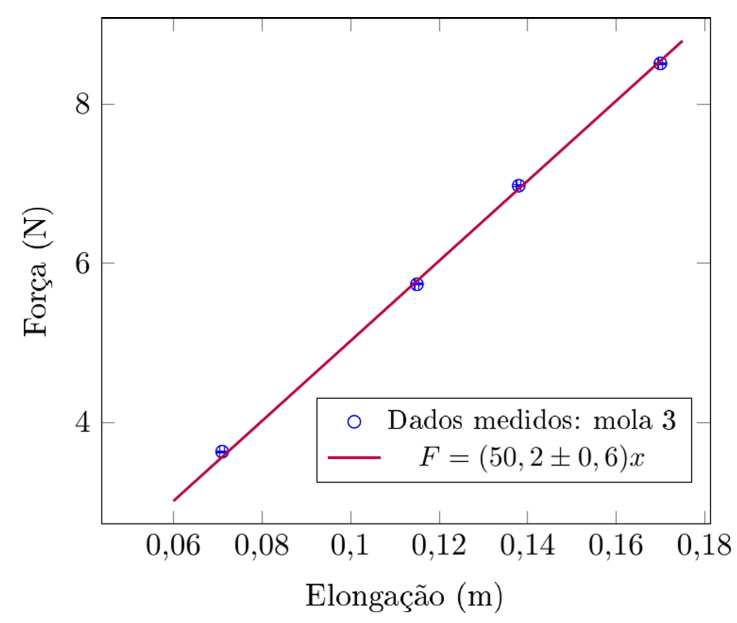

Figura 6: Constante de Rigidez da Mola 3

o cálculo das seguintes grandezas: frequências dos modos de vibração (autovalores), razões de amplitude (autovetores), amplitudes da massa 1 no primeiro e no segundo modo, ângulos de fase e posição como função do tempo para $m_{1}$ e $m_{2}$. As massas e as constantes elásticas foram informadas conforme as medidas, descritas na seção 3 . Os valores das massas e constantes usadas no Modellus são as seguintes:

$$
\begin{array}{ll}
\text { - } & m_{1}=0,341 \mathrm{~kg} \\
\text { - } & m_{2}=0,624 \\
\text { - } & k_{1}=132,3 \\
\text { - } & k_{2}=59,4 \\
\text { - } & k_{3}=50,2
\end{array}
$$

Utilizando os valores de massa e constantes elásticas previamente apresentados, foram calculadas as duas frequências naturais, por meio da equação (11). Para o primeiro modo de vibração a frequência é de 11,69 $\pm 0,05$ $\mathrm{rad} / \mathrm{s}$ e para o segundo modo de vibração a frequência é de 24,52 $\pm 0,07 \mathrm{rad} / \mathrm{s}$. Também foram calculados os autovetores, sendo os valores obtidos de $r_{1}=2,44$ e $r_{2}=$ $-0,22$. As incertezas no cálculo das frequências naturais 
foram realizadas pelo método das derivadas parciais de acordo com $[15$.

Para as simulações com o Modellus é necessário definir as condições iniciais do sistema, que são as velocidades $\left(v_{1}\right.$ e $\left.v_{2}\right)$ e posições de cada massa $\left(x_{1}\right.$ e $\left.x_{2}\right)$ no tempo $t=0$. De posse das condições inicias, o software calcula (através das equações de entrada digitadas) as grandezas necessárias para a plotagem do gráfico de movimento e cria uma animação. Uma tabela com as variáveis calculadas em cada passo de tempo também é apresentada.

$\mathrm{Na}$ animação as massas foram representadas por blocos retangulares, com cores diferentes e com molas de tamanho variável acompanhando a vibração de cada massa. Isso permite uma melhor comparação com o projeto real. Além disso, são gerados gráficos mostrando a variação da amplitude de movimento de cada massa ao longo do tempo. No gráfico gerado pelo Modellus, as curvas de movimento levam a mesma cor da sua respectiva massa. A interface do programa é mostrada na Fig. 7

Na Fig. 8 são mostradas as curvas de posição $\times$ tempo de cada massa para o primeiro modo de vibração, geradas pelo Modellus . Para este modo de vibração, as condições iniciais usadas são: $v_{1}=0 \mathrm{~m} / \mathrm{s}, v_{2}=0 \mathrm{~m} / \mathrm{s}, x_{1}=0,01 \mathrm{~m}$ e $x_{2}=r_{1} x_{1}=0,0244 \mathrm{~m}$. Pode-se verificar que neste caso as massas oscilantes possuem mesma frequência, estão em fase e apresentam amplitudes diferentes (relacionadas por $\left.r_{1}\right)$.

A Fig. 9 mostra as curvas de posição em função do tempo para o sistema vibrando no segundo modo de vibração. Para este modo, as condições iniciais usadas são: $v_{1}=0 \mathrm{~m} / \mathrm{s}, v_{2}=0 \mathrm{~m} / \mathrm{s}, x_{1}=0,01 \mathrm{~m}, x_{2}=r_{2} x_{1}=$ $-0,022 \mathrm{~m}$. Verifica-se que nesta situação as massas oscilantes também possuem mesma frequência, porém estão defasadas de $180^{\circ}$ e também apresentam amplitudes diferentes (relacionadas por $r_{2}$ ).

Foi também realizada uma simulação do sistema operando em uma situação que não no primeiro ou segundo modo de vibração (Fig. 10). Para que o sistema opere dessa forma, basta que as condições iniciais não sejam àquelas de velocidade inicial igual a zero e que as posições iniciais não tenham a relação $x_{2}=r_{1} x_{1}$ para o primeiro modo de vibração ou $x_{2}=r_{2} x_{2}$ para o segundo modo de vibração. No caso apresentado na Fig. 10 as condições iniciais usadas foram $v_{1}=0 \mathrm{~m} / \mathrm{s}, v_{2}=\mathrm{m} / \mathrm{s}, x_{1}=0 \mathrm{~m}$, $x_{2}=0,09 \mathrm{~m}$. Nota-se que neste caso as massas oscilam influenciadas por ambos os modos de vibração ao mesmo tempo, cujo resultado depende das condições iniciais.

\section{Análise do sistema vibratório usando o Tracker}

O Tracker é um software livre que permite a análise de movimento via análise de vídeo. Para este trabalho, os vídeos foram feitos com uma câmera Canon modelo 60D acoplada a uma objetiva Canon 10:22 mm e filmados a uma taxa de aquisição de 60 qps (quadros por segundo) em foco manual. Para este estudo, gravar com uma taxa elevada de qps é de fundamental importância, visto que resulta em uma maior precisão na obtenção de dados. Sendo 60 qps a taxa de aquisição, obtém-se 60 valores de posição de cada massa para cada segundo gravado em vídeo.

É importante que a taxa de aquisição do sistema de aquisição de dados (no caso a taxa de qps) atenda o critério de Nyquist. De acordo com o critério de Nyquist [16], uma taxa de aquisição é suficiente para que seja possível reconstruir um sinal medido desde que a maior frequência envolvida na oscilação não supere a metade da taxa de aquisição. Como, no caso estudado neste trabalho, a maior frequência envolvida na oscilação é de $24,52 \mathrm{~Hz}$ e a taxa de aquisição é de $60 \mathrm{~Hz}$, então o critério de Nyquist foi respeitado.

Como um dos objetivos deste trabalho é comparar os dados simulados no Modellus com os obtidos pela análise via Tracker, também é necessária uma boa resolução do vídeo, pois o software Tracker obtém os dados através do próprio vídeo. Para tanto, a gravação foi realizada com uma resolução de 640 x 480 pixels, sendo essa suficiente para que o software consiga distinguir o fundo do vídeo das massas oscilantes. Para facilitar a identificação das massas pelo software Tracker, o ambiente de gravação escolhido tem fundo claro e foram adicionados pontos coloridos de $10 \mathrm{~mm}$ de diâmetro às massas, facilitando a identificação realizada pelo software. A Fig. 11 a) ilustra a interface do Tracker, que obteve uma boa aproximação da vibração da massa 2 para o primeiro mode de vibração. Já na Fig. 11b) é ilustrado o uso do software Grapher 17], que, através dos dados obtidos pelo Tracker, consegue uma melhor aproximação da oscilação real da massa 2 no primeiro modo de vibração.

Ao confrontar os resultados obtidos pela análise realizada com o Tracker com os valores calculados pelo Modellus, observa-se uma pequena diferença nas frequências.

Para o modo 1, a frequência de oscilação das massas calculada pelo Modellus é de 11,69 rad/s e para o segundo modo 24,52 rad/s. Utilizando os dados obtidos pelo Tracker e utilizando o software Curver Expert, que ajusta os parâmetros do modelo físico desejado, obteve-se $12,86 \mathrm{rad} / \mathrm{s}$ para o primeiro modo de vibração.

Fez-se também a análise das vibrações por espectro de frequência, usando Transformada Rápida de Fourier (FFT) por meio do software Sigview 18], com o objetivo de confirmar os valores de frequências por outro método. Utilizar uma FFT como ferramenta de análise em um sistema vibracional possibilita uma análise no domínio de frequência ao invés do domínio de tempo.

A Fig. 12 apresenta os picos de frequência para a massa 1 e a Fig. 13 apresenta os picos de frequência para a massa 2, em ambos os casos, para o sistema tendo sido posto a oscilar com condições iniciais necessárias para o primeiro modo de vibração.

Na análise de FFT para a massa 1, na tentativa de por o sistema a oscilar no primeiro modo de vibração, foram 
encontrados duas frequências de amplitudes significativas, $2,16 \mathrm{~Hz}(13,57 \mathrm{rad} / \mathrm{s})$ de amplitude igual a 4,8 $\mathrm{mm}$ e $4,32 \mathrm{~Hz}(27,14 \mathrm{rad} / \mathrm{s})$ de amplitude 1,1 mm. Neste caso,

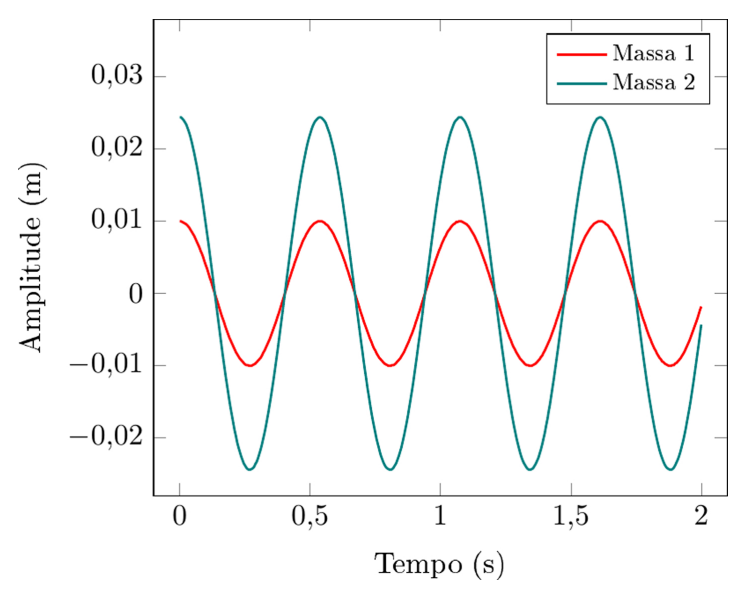

Figura 8: Modo de vibração 1

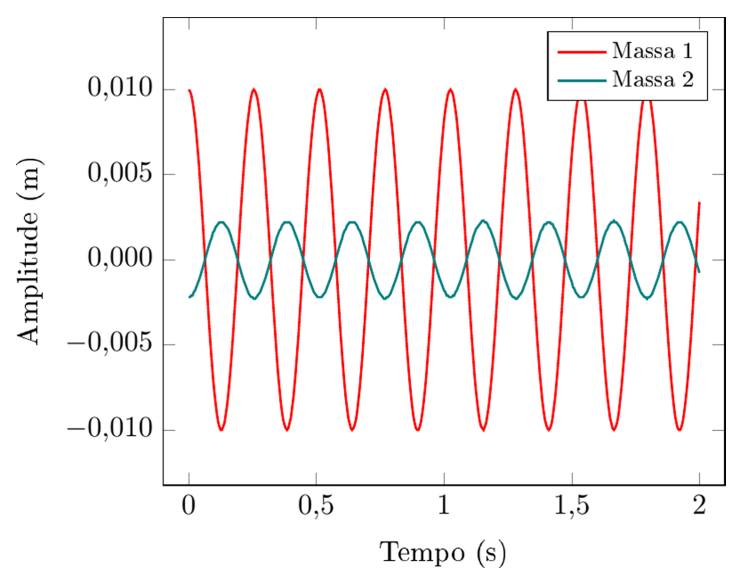

Figura 9: Modo de vibração 2

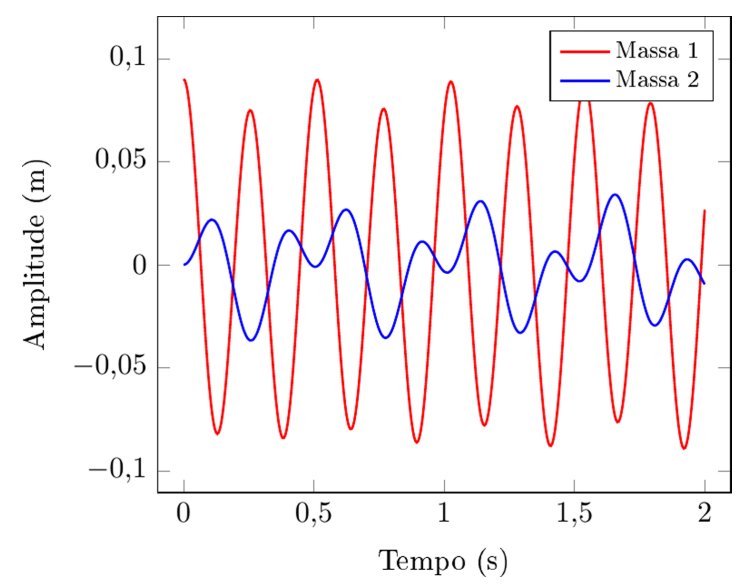

Figura 10: Sistema vibrando em ambos os modos de vibração ao mesmo tempo

verifica-se claramente que a massa 1 estava oscilando com uma influência do segundo modo de vibração, ou seja, não estava totalmente no primeiro modo de vibração. Isto ocorre, pois a condição inicial imposta ao sistema não satisfez exatamente o necessário para o sistema vibrar integralmente no primeiro modo de vibração.

Já para a massa 2 foram encontradas $2,16 \mathrm{~Hz}$ com amplitude de 11,5 mm e 4,32 $\mathrm{Hz}$ de amplitude 0,2 mm. Nesse caso, a influência da segunda frequência natural na vibração da massa é muito baixa.

Tanto na Fig. 12 como na Fig. 13 o software utilizado para aplicar a FFT aponta os cinco maiores picos de frequência. É possível observar, no caso da Fig. 12 que existem dois picos com amplitudes significativas, correspondentes as duas frequências naturais do sistema. Os demais picos podem ser interpretados como modos de vibração perpendicular ao campo gravitacional, já que é possível que as massas se movam nessa direção, embora isso não seja desejável no experimento. Também, os
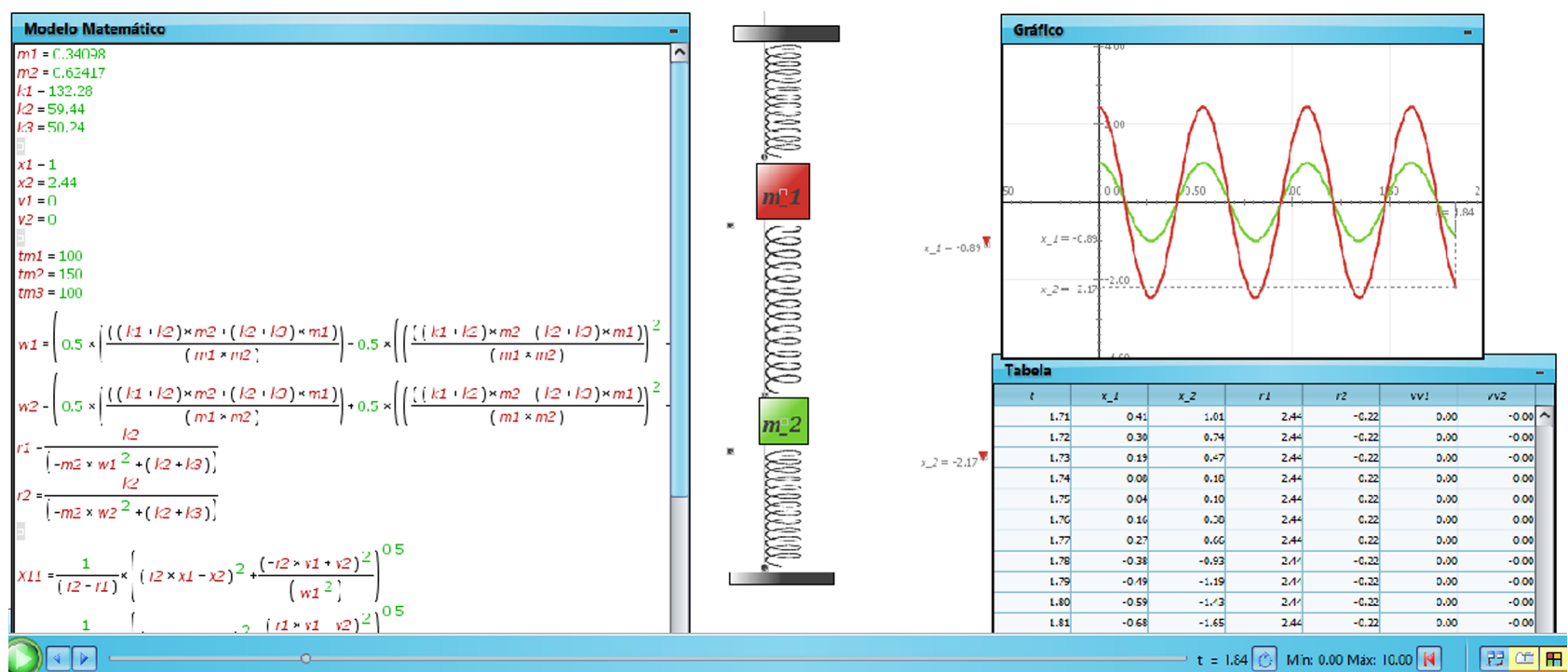

Figura 7: Interface do programa Modellus. 


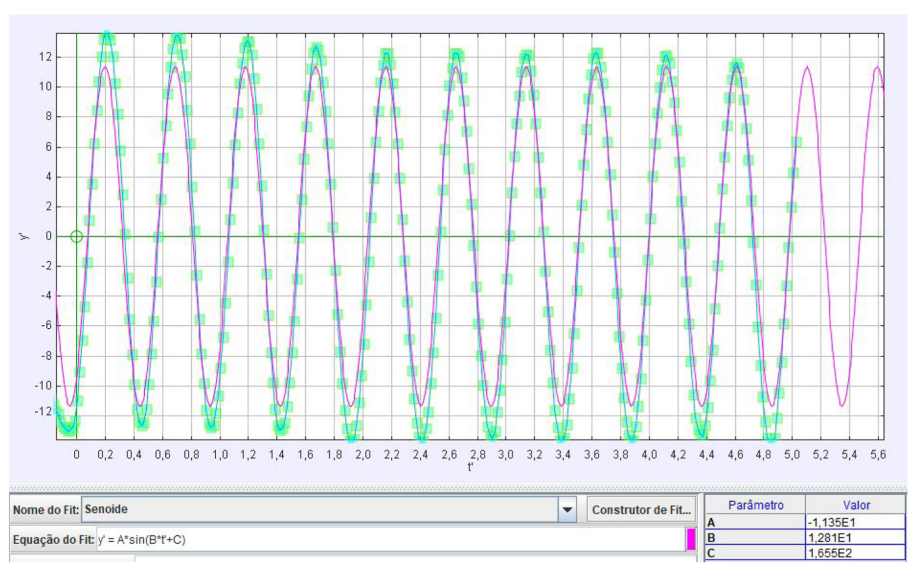

(a)

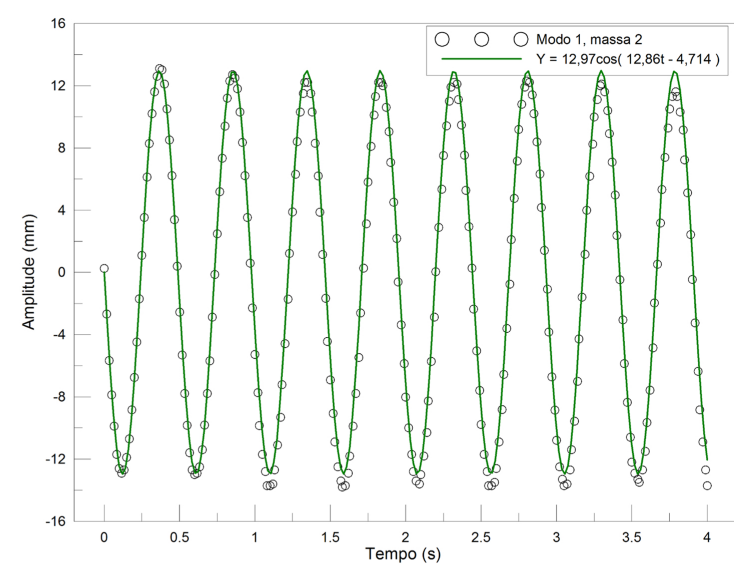

(b)

Figura 11: Oscilação da massa 2 no primeiro modo de vibração (a) Tracker e (b) Grapher.

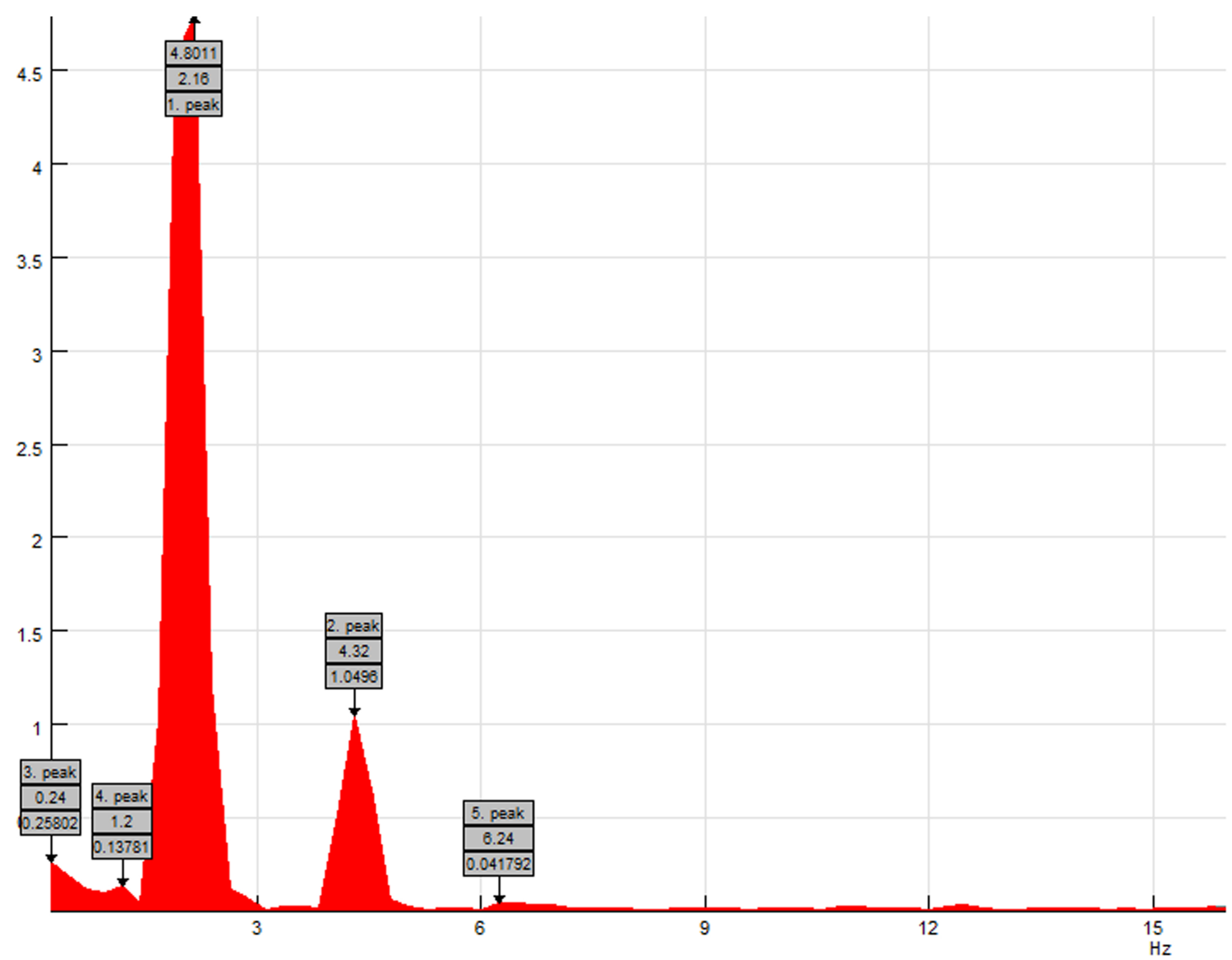

Figura 12: Picos de frequência para o primeiro modo de vibração para a massa 1 .

demais picos de frequência podem ser atribuídos a limitações da FFT que obtém melhor resposta em reproduzir a oscilação original adicionando pequenas amplitudes com outras frequências. O mesmo raciocínio se aplica a Fig. 13.

Foi encontrada uma diferença de aproximadamente $10 \%$, entre o valor teórico e o obtido pelo ajuste dos dados com o uso do software Curve Expert. Aplicando a FFT, a diferença com relação ao valor teórico ficou em aproximadamente $16 \%$. 


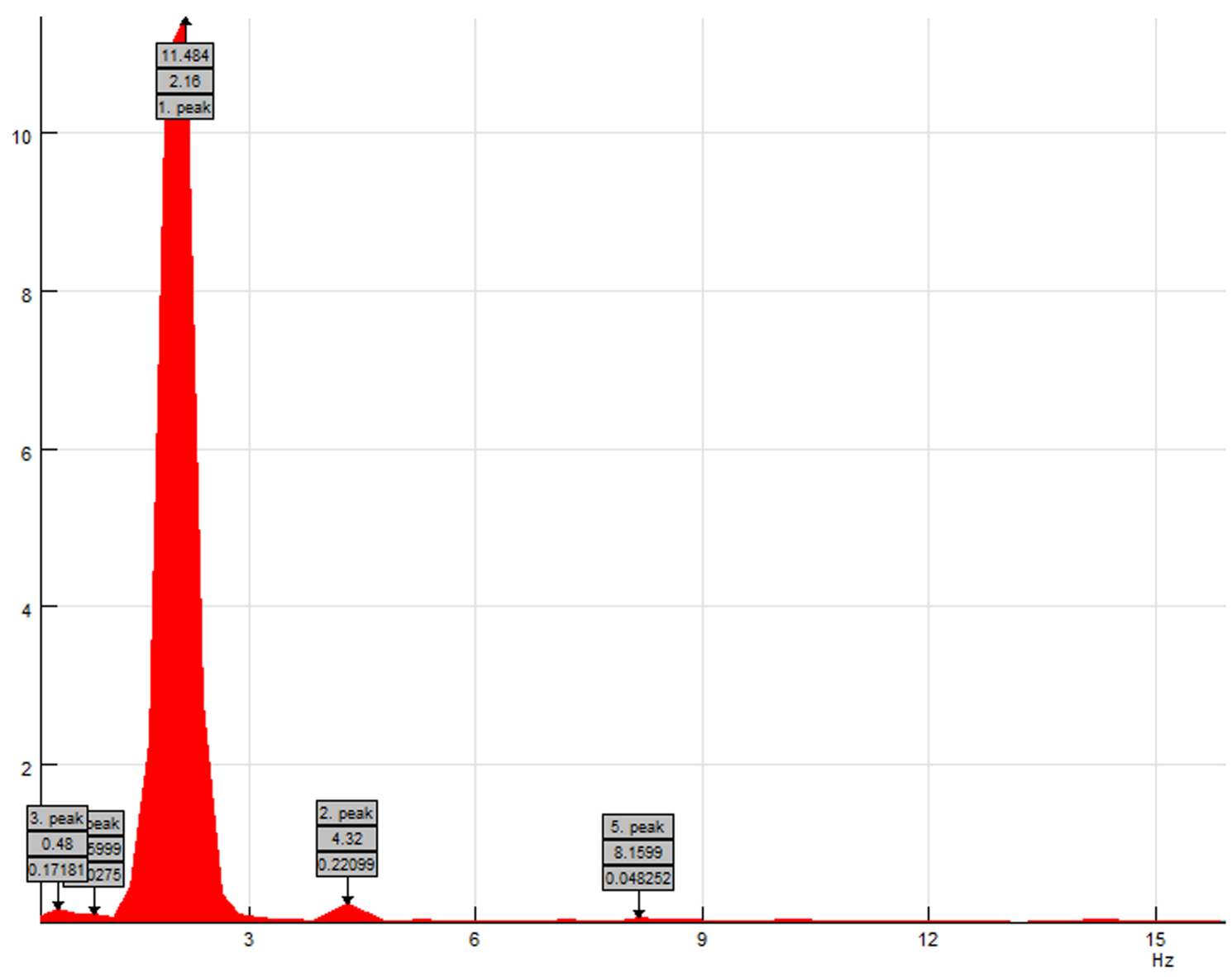

Figura 13: Picos de frequência para o primeiro modo de vibração para a massa 2.

\section{Conclusões}

Este trabalho apresentou uma abordagem teórico-experimental para o estudo de sistemas vibracionais com dois graus de liberdade. Um sistema real foi construido usando duas massas de valores diferentes e três molas com constantes elásticas distintas. O sistema foi simulado no software Modellus fornecendo os dois modos de vibração em 11,69 rad/s e 24,52 rad/s.

A oscilação do sistema vibratório foi analisada por meio de dados coletados com uso do software Tracker. O sistema foi colocado a vibrar com condições iniciais que são necessárias para o primeiro modo de vibração. Essas condições são velocidade inicial igual a zero para ambas as massas e posições iniciais iguais a $x_{1}(0)$ e $x_{2}(0)=r_{1} x_{1}(0)$. Utilizando o software Curver Expert foram encontrados os parâmetros de melhor ajuste para a curva teórica. A tentativa de por o sistema a vibrar no primeiro modo de vibração forneceu uma frequência de 12,86 rad/s. Aplicando uma FFT foi encontrada uma frequência de $13,57 \mathrm{rad} / \mathrm{s}$.

A diferença entre os resultados obtidos pelos métodos de análises se deve principalmente ao fato de que a equação usada para ajuste no Curver Expert supõem que o sistema está vibrando totalmente no primeiro modo, e assim, só há uma frequência atuando no sistema. Entre- tanto, observando a FFT, nota-se que ambos os modos de vibração estão operando, embora a influência da segunda frequência natural seja bem menor do que a primeira. Isso confirma que o esforço por colocar o sistema a vibrar no primeiro modo foi praticamente atingido.

As diferenças encontradas entre os dois métodos de análise e o resultado teórico (10\% para o ajuste da curva teórica e $16 \%$ para a FFT) podem ser explicadas por erros experimentais relativos às medidas e eventuais perturbações indesejadas na vibração, como por exemplo, pequenas amplitudes de vibração perpendicular ao eixo das molas.

Não foram feitas análises para o segundo modo de vibração, pois não foi possível por o sistema a oscilar neste modo manualmente. Ocorre que para o caso estudado neste trabalho a posição inicial conferida a $m_{2}$ deve ser igual a $-0,22$ vezes a posição conferida a $m_{1}$.

Por fim pode-se concluir que atividades como a proposta neste trabalho, envolvendo a análise teórica de um fenômeno físico, construção de um protótipo para teste e análises matemáticas é uma excelente alternativa de ensino de temas de Engenharia e Física. Também, é interessante ressaltar que com o uso de softwares como o Tracker, experimentos na área de vibrações mecânicas podem ser realizados com pouco investimento provendo 
bons resultados, principalmente para finalidades didáticas.

\section{Referências}

[1] M. Varanis, A.L. Silva, P.H.A. Brunetto e R.F. Gregolin, Rev. Bras. Ensino Fís. 38, 1301 (2016).

[2] M. Varanis, A.L. Silva e A.G. Mereles, Rev. Bras. Ensino Fís. 40, e1304 (2018).

[3] J.M. Mahoney e R. Nathan, Mechanical Vibrations Modal Analysis Project with Arduinos, disponível em https://www.asee.org/public/conferences/ 78/papers/18885/view.

[4] W. Bonventi Jr. e N. Aranha, Rev. Bras. Ensino Fís. 37, 2504 (2015).

[5] http://cietenped.ufscar.br/submissao/index.php/ 2018/article/view/127

[6] S.S. Rao e F.F. Yap, Mechanical Vibrations (AddisonWesley, Boston, 1995), $3^{a}$ ed.

[7] Curve Expert, acessado em 13/10/2018: https://www. curveexpert.net/products/curveexpert-basic/

[8] D. Halliday, R. Resnick e J. Walker, Fundamentos de Física: Gravitação, Ondas e Termodinâmica (Editora LTC, Rio de Janeiro, 2016), v. 2, p. 372.

[9] P.A. Tipler e G. Mosca, Física para Cientistas e Engenheiros (Editora LTC, Rio de Janeiro, 2009), v. 1, p. 788.

[10] V.M. Nishi Ueta, Análise de um sistema de vibração com três graus de liberdade: Comparação entre três métodos de solução. Projeto de Graduação, Universidade Federal do Rio de Janeiro, Rio de Janeiro (2015).

[11] F.L. Silveira, Rev. Bras. Ensino Fís. 37, 2306 (2015).

[12] J.F. Mendes, I.F. Costa e C.M.S.G. de Sousa, Rev. Bras. Ensino Fís. 34, 2402 (2012).

[13] I.S. Araujo, Simulação e Modelagem Computacional como Recursos Auxiliares no Ensino de Física Geral. Tese de Doutorado, Universidade Federal do Rio Grande do Sul, Rio Grande do Sul (2005).

[14] D.C.M. Torresan, O Uso do Software de Simulação Modellus na Conceitualização de Derivada: Experiências de Ensino-Aprendizagem com base em Vergnaud. Dissertação de Mestrado, Universidade Luterana do Brasil, Rio Grande do Sul (2008).

[15] J.R. Taylor, Introdução à análise de erros - o estudo de incertezas em mediações físicas (Editora Bookman, Porto Alegre ,2012), $2^{a}$ ed.

[16] C.E. Shannon, Proceedings of the IRE 37, 10 (1949).

[17] Grapher, acesso em 13/10/2018: https://www. goldensoftware.com/products/grapher.

[18] Sigview, acesso em 13/10/2018: http://https://www. sigview.com 